\title{
Perfil epidemiológico de idosos que foram a óbito por queda no Rio Grande do Sul
}

\author{
Epidemiologic profile of elderly people who died because of falls in Rio Grande do Sul state, Brazil
}

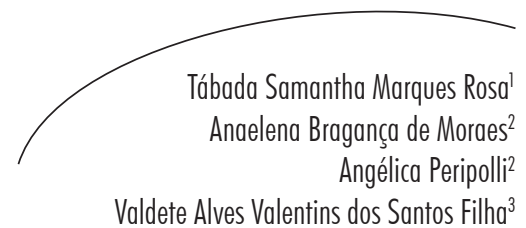

Resumo

Objetivo: Traçar o perfil dos idosos que foram a óbito por queda no Rio Grande do Sul no período de 2006 a 2011. Métodos: Foram analisados 2.126 óbitos por queda em idosos no RS de 2006 a 2011, registrados no Sistema de Informações sobre Mortalidade. As análises estatísticas foram realizadas utilizando-se o aplicativo computacional PASW 17.0. Resultados: A chance de óbitos por queda nos idosos é significativamente maior para o gênero feminino, para a faixa etária acima de 69 anos, para idosos com cor de pele branca, para viúvos ou solteiros. Houve acréscimo de $41,8 \%$ nos coeficientes de mortalidade específicos por queda no período do estudo, o maior coeficiente ocorrendo em 2011 (31,56 óbitos por queda a cada 100 mil idosos), maior para o gênero feminino e para idade de 80 anos ou mais. Conclusão: Constatou-se que o coeficiente de mortalidade por queda aumentou de 2006 para 2011 naquele estado, sendo mais elevado para os idosos de 80 anos ou mais, resultados de relevância para o desenvolvimento de políticas públicas para o idoso.

\section{Abstract}

Objective: To determine the characteristics of elderly who died by falling in Rio Grande do Sul state, Brazil, from 2006 to 2011. Methods: We analyzed 2,126 deaths from falls in the state from 2006 to 2011, registered in the Brazilian Mortality Information System. Statistical analyzes were performed using the SPSS 17.0 computer application. Results: The chance of death from falls in the elderly is significantly higher for females, age group above 69 years and elderly people with white skin color, widowed or single. There was a $41.8 \%$ increase in specific mortality rates fall during the study period, the highest rate occurring in 2011 (31.56 deaths fall among 100,000 elderly), higher for females and age 80 years or more. Conclusion: It was found that the mortality rate from falls increased from 2006 to 2011 in that state, being highest for those aged 80 and over, relevance of results for the development of public policies for the elderly.

\footnotetext{
Universidade Federal de Santa Maria, Centro de Ciências da Saúde, Programa de Pós-graduação em Distúrbios da Comunicação Humana, Departamento de Fonoaudiologia. Santa Maria, RS, Brasil.

2 Universidade Federal de Santa Maria, Centro de Ciências Naturais e Exatas, Departamento de Estatística. Santa Maria, RS, Brasil.

3 Universidade Federal de Santa Maria, Centro de Ciências da Saúde, Departamento de Fonoaudiologia. Santa Maria, RS, Brasil.
}

\section{Palavras-chave:}

Envelhecimento. Acidentes por Quedas. Coeficiente de Mortalidade.

Key words: Aging. Accidental Falls. Mortality Rate. 


\section{INTRODUÇÃO}

O aumento da população de idosos é uma realidade mundial. ${ }^{1}$ Isto é destacado pela demografia como um sinal de desenvolvimento e para a saúde pública é uma conquista, pois desejar vida longa é uma ambição de todo ser humano. ${ }^{2}$ Nos últimos anos, os padrões de mortalidade da população idosa, em países desenvolvidos e em desenvolvimento, têm se mantido semelhantes. ${ }^{3}$

O crescimento do número de pessoas com 60 anos ou mais no Brasil vem aumentando. Em 1960, 3,3 milhões de brasileiros eram idosos e representavam $4,7 \%$ da população. Em 2000, 14,5 milhões, ou 8,5\% dos brasileiros, estavam nessa faixa etária. Já em 2010, passou de 10,8\% da população, ou seja, 20,5 milhões de idosos. ${ }^{4}$ Destaca-se, ainda, que o Brasil está entre os países da América Latina com o maior aumento no número de pessoas idosas. ${ }^{5}$ A população do Rio Grande do Sul (RS) também tem apresentado acelerado processo de envelhecimento populacional e com um padrão diferenciado de morbi-mortalidade para as faixas etárias acima de 60 anos. Isso ocorre porque o RS é um dos estados com maior índice de desenvolvimento humano, maior expectativa de vida e de proporção de idosos na população. ${ }^{6}$

Vários estudos nacionais ${ }^{7,8}$ e internacionais ${ }^{9,10}$ citam as quedas como importante causa de mortalidade, morbidade e incapacitações entre a população idosa. A queda pode ser definida como um evento não intencional que tem como resultado a mudança da posição inicial do indivíduo para um mesmo nível ou nível mais baixo. $^{11}$

Com o envelhecimento, o corpo humano entra em processo de declínio fisiológico, com a diminuição da densidade óssea e da massa muscular, instabilidade postural, ${ }^{12,13}$ comprometimento da capacidade visual e auditiva, maior consumo de medicamentos devido à presença de inúmeras doenças comuns ao idoso, ${ }^{14}$ além de riscos ambientais que podem predispor à queda. ${ }^{5}$ Estudo de base populacional verificou que a prevalência de quedas e quedas recorrentes em idosos foram, respectivamente, $37,5 \%$ e $16,5 \% .^{15}$

No que diz respeito às circunstâncias dessas ocorrências, autores apontam que grande parte das quedas ocorrem na própria residência do idoso. ${ }^{8,15}$ No Brasil, ainda são poucos os programas que focam a prevenção de quedas, diferentemente do que ocorre em países como Canadá, Austrália, França e Estados Unidos. Pesquisas sobre as características do idoso que cai são importantes para que sejam traçados programas que tenham foco nesta prevenção. ${ }^{16,17}$

Em relação às lesões determinadas pelas quedas, as fraturas de fêmur são a causa mais frequente de internações e emergências, sendo que $30 \%$ dos idosos vão a óbito em até um ano. ${ }^{8,18}$ Salienta-se, ainda, que entre os idosos residentes no município de Campinas-SP que tiveram quedas relatadas como principal acidente sofrido nos últimos 12 meses, 56,7\% tiveram suas atividades diárias limitadas, 58,6\% ficaram acamados e 71,2\% receberam assistência médica em decorrência da queda. ${ }^{19}$

Mesmo quando as quedas causam lesões menores, elas podem afetar a qualidade de vida dos idosos, levando ao medo de cair com consequente restrição de atividades, mobilidade, diminuição da atividade física, isolamento social e depressão. ${ }^{20,21}$ Desta forma, as quedas têm expressiva predominância entre os fatores externos de ferimentos não intencionais, sendo codificadas como W00-W19 na Classificação 
Internacional de Doenças - Décima Revisão (CID-10), ${ }^{22}$ que inclui um amplo leque de quedas, abrangendo inclusive as que ocorrem no mesmo nível, de nível mais alto e outras quedas não especificadas.

Frente ao panorama observado e aos poucos estudos encontrados sobre mortalidade por queda em idosos, verifica-se a importância da realização deste estudo que teve como objetivo traçar o perfil dos idosos que foram a óbito por queda, no Rio Grande do Sul, no período de 2006 a 2011. Visa-se proporcionar, assim, mais subsídios para o desenvolvimento de políticas públicas adequadas que atendam às demandas específicas para essa faixa etária no referido estado.

\section{METODOLOGIA}

Trata-se de estudo descritivo de série temporal baseado em dados secundários oficiais sobre os óbitos da população brasileira com 60 anos ou mais de idade associados à queda, registrados no Sistema de Informações sobre Mortalidade (SIM), ${ }^{23}$ composto pelas informações provenientes das declarações de óbito e disponibilizado pelo Departamento de Informática do SUS (DATASUS). ${ }^{24}$ Para a definição da população idosa, optou-se por estudar a faixa etária de 60 anos ou mais, idade limite ou inicial do envelhecimento estabelecida pela Política Nacional do Idoso. ${ }^{25}$

Foram consideradas as informações sobre gênero, idade, cor da pele, estado civil e escolaridade, relativas à população de idosos residentes no estado do RS que foram a óbito no período de 2006 a 2011 que constam na Declaração de Óbito (DO) e são registradas no SIM.

Os idosos que foram a óbito por queda foram selecionados pela identificação dos códigos referentes à CID-10, as chamadas causas externas de morbidade e mortalidade. Foram selecionados os óbitos codificados entre W00 e W19, pertencentes à categoria "quedas". Posteriormente, foram realizadas algumas adaptações no banco de dados, tais como: a junção das informações anuais em um único arquivo e a eliminação dos indivíduos que não continham a informação da idade, pela relevância dessa informação para a definição do banco de dados. O banco de dados ficou constituído de 309.840 idosos que foram a óbito, sendo 2.126 por queda.

Os dados da população idosa residente no RS para a construção dos coeficientes de mortalidade específicos foram obtidos por meio do site do DATASUS/MS. Como indicador de mortalidade por causa externa, foi utilizado o coeficiente de mortalidade específico por queda, calculado por ano, gênero e faixa etária.

As análises estatísticas foram realizadas utilizando-se o aplicativo computacional PASW 17.0. Foram calculadas as razões de chance $(O R)$ e seus respectivos intervalos de confiança de $95 \%$. Como os bancos de dados do SIM são de domínio público, sem identificação nominal, não houve necessidade de submissão deste estudo a um Comitê de Ética em Pesquisa.

\section{RESULTADOS}

Dos 309.840 óbitos de idosos, 2.126 apresentaram como causa básica a queda, o que corresponde a $0,69 \%$ das causas de óbito no período pesquisado no estado do RS.

Na tabela 1, são apresentadas as frequências absolutas e os percentuais dos óbitos por queda e o total de óbitos segundo gênero, faixas de idade (anos), cor da pele, estado civil e escolaridade, bem como os valores da razão de chances $(O R)$ e seus respectivos intervalos de confiança de $95 \%$. 
Tabela 1. Distribuição de frequências dos óbitos por queda, total e OR das covariáveis para os idosos no RS, no período de 2006 a 2011.

\begin{tabular}{|c|c|c|c|c|c|}
\hline Variável & Categorias & $\begin{array}{l}\text { Óbitos por } \\
\text { queda (\%) }\end{array}$ & $\%$ & $\begin{array}{r}\text { Total de } \\
\text { óbitos }\end{array}$ & $\mathrm{OR}^{*}(\mathrm{IC} 95 \%)$ \\
\hline \multirow{3}{*}{ Gênero } & Masculino & $917(0,60)$ & 43,1 & 154.039 & 1 \\
\hline & Feminino & $1.209(0,78)$ & 56,9 & 155.801 & $1,31(1,20 ; 1,42)$ \\
\hline & Total & 2126 & 100,0 & 309.840 & - \\
\hline \multirow{4}{*}{$\begin{array}{l}\text { Faixas de } \\
\text { idade (anos) }\end{array}$} & De 60 a 69 & $344(0,43)$ & 16,2 & 80.438 & 1 \\
\hline & De 70 a 79 & $557(0,53)$ & 22,2 & 105.664 & $1,23(1,08 ; 1,41)$ \\
\hline & 80 ou mais & $1.225(0,99)$ & 57,6 & 123.738 & $2,33(2,07 ; 2,62)$ \\
\hline & Total & 2126 & 100,0 & 309.840 & - \\
\hline \multirow{6}{*}{ Cor da pele } & Branca & $2.000(0,72)$ & 95,5 & 278.421 & $2,00(1,52 ; 2,63)$ \\
\hline & Preta & $52(0,36)$ & 2,5 & 14.424 & 1 \\
\hline & Amarela & $3(0,94)$ & 0,1 & 318 & $2,63(0,82 ; 8,47)$ \\
\hline & Parda & $38(0,38)$ & 1,8 & 10.078 & $1,05(0,69 ; 1,59)$ \\
\hline & Indígena & $2(0,71)$ & 0,1 & 283 & $1,97(0,48 ; 8,12)$ \\
\hline & Total & 2095 & 100,0 & 303.525 & - \\
\hline \multirow{6}{*}{ Estado civil } & Solteiro & $261(0,68)$ & 12,8 & 38.612 & $1,16(1,01 ; 1,34)$ \\
\hline & Casado & $702(0,58)$ & 34,3 & 120.296 & 1 \\
\hline & Viúvo & $991(0,82)$ & 48,5 & 120.263 & $1,42(1,28 ; 1,56)$ \\
\hline & Separado** & $90(0,58)$ & 4,4 & 15.468 & $1,00(0,80 ; 1,24)$ \\
\hline & Outro & $0(0)$ & 0,0 & 569 & - \\
\hline & Total & 2044 & 100,0 & 295.208 & - \\
\hline \multirow{6}{*}{$\begin{array}{l}\text { Escolaridade (anos } \\
\text { de estudo) }\end{array}$} & Nenhum & $181(0,55)$ & 15,7 & 33.676 & $0,98(0,82 ; 1,17)$ \\
\hline & 1 a 3 & $467(0,62)$ & 40,6 & 75.369 & $1,13(0,98 ; 1,30)$ \\
\hline & 4 a 7 & $336(0,55)$ & 29,2 & 61.259 & 1 \\
\hline & 8 a 11 & $99(0,59)$ & 8,6 & 16.691 & $1,08(0,86 ; 1,35)$ \\
\hline & 12 e mais & $67(0,68)$ & 5,8 & 9.812 & $1,25(1,96 ; 1,62)$ \\
\hline & Total & 1150 & 100,0 & 196.807 & - \\
\hline Total geral & & $2.126(0,69)$ & & 309.840 & \\
\hline
\end{tabular}

Dados faltantes (óbito por queda; óbito total): cor da pele (31; 6.316); estado civil (82; 14.633); escolaridade (976; 113.033); *Odds ratio; **Separado judicialmente.

Considerando a razão de chances, verificase que a chance de óbitos por queda nos idosos é significativamente maior para o gênero feminino, para a faixa etária acima de 69 anos, para idosos com cor de pele branca, seguidos dos viúvos e solteiros. Para a população avaliada, a escolaridade não esteve associada com a ocorrência de óbitos por quedas.
O número de idosos na população, o incremento percentual da população idosa, as frequências de óbitos por queda, bem como os coeficientes de mortalidade específicos por queda na população idosa, são apresentados na tabela 2. 
Tabela 2. Número e incremento percentual de idosos na população, mortalidade por queda e coeficiente de mortalidade específico por queda (por 100 mil idosos), no RS de 2006 a 2011.

\begin{tabular}{cccccc}
\hline \multirow{2}{*}{ Ano } & \multirow{2}{*}{$\begin{array}{c}\text { No de } \\
\text { idosos }\end{array}$} & Incremento & \multicolumn{2}{c}{ Quedas } & CME \\
\cline { 4 - 5 } & $(\%)$ & Não $(\%)$ & Sim $(\%)$ & por queda \\
\hline 2006 & 1.136 .710 & Base* & $47.790(99,47)$ & $253(0,53)$ & 22,26 \\
2007 & 1.350 .294 & 18,87 & $50.408(99,34)$ & $336(0,66)$ & 24,88 \\
2008 & 1.367 .604 & 20,31 & $49.138(99,27)$ & $359(0,73)$ & 26,25 \\
2009 & 1.416 .830 & 24,64 & $51.708(99,32)$ & $354(0,68)$ & 24,99 \\
2010 & 1.459 .597 & 28,40 & $53.314(99,32)$ & $362(0,68)$ & 24,80 \\
2011 & 1.463 .813 & 28,78 & $55.457(99,17)$ & $462(0,83)$ & 31,56 \\
\hline Total & 8.194 .848 & - & $307.815(99,31)$ & $2.126(0,69)$ & 25,94 \\
\hline
\end{tabular}

* Ano base; $\mathrm{CME}=$ Coeficiente de mortalidade específico.

Pode-seobservarque em relação ao incremento percentual do número de idosos na população do RS, houve aumento progressivo de $28,78 \%$ de 2006 para 2011. Esses resultados se refletem nos coeficientes de mortalidade específicos por queda, que também apresentaram acréscimo, porém de $41,8 \%$ no período do estudo. Ressaltase que o maior coeficiente ocorreu em 2011 e foi de 31,56 óbitos por queda a cada 100 mil idosos. É importante observar que o aumento do valor desse coeficiente foi maior do que o aumento do número de idosos na população.

Os coeficientes de mortalidade específicos por queda e gênero, de 2006 a 2011 estão representados na figura 1 .

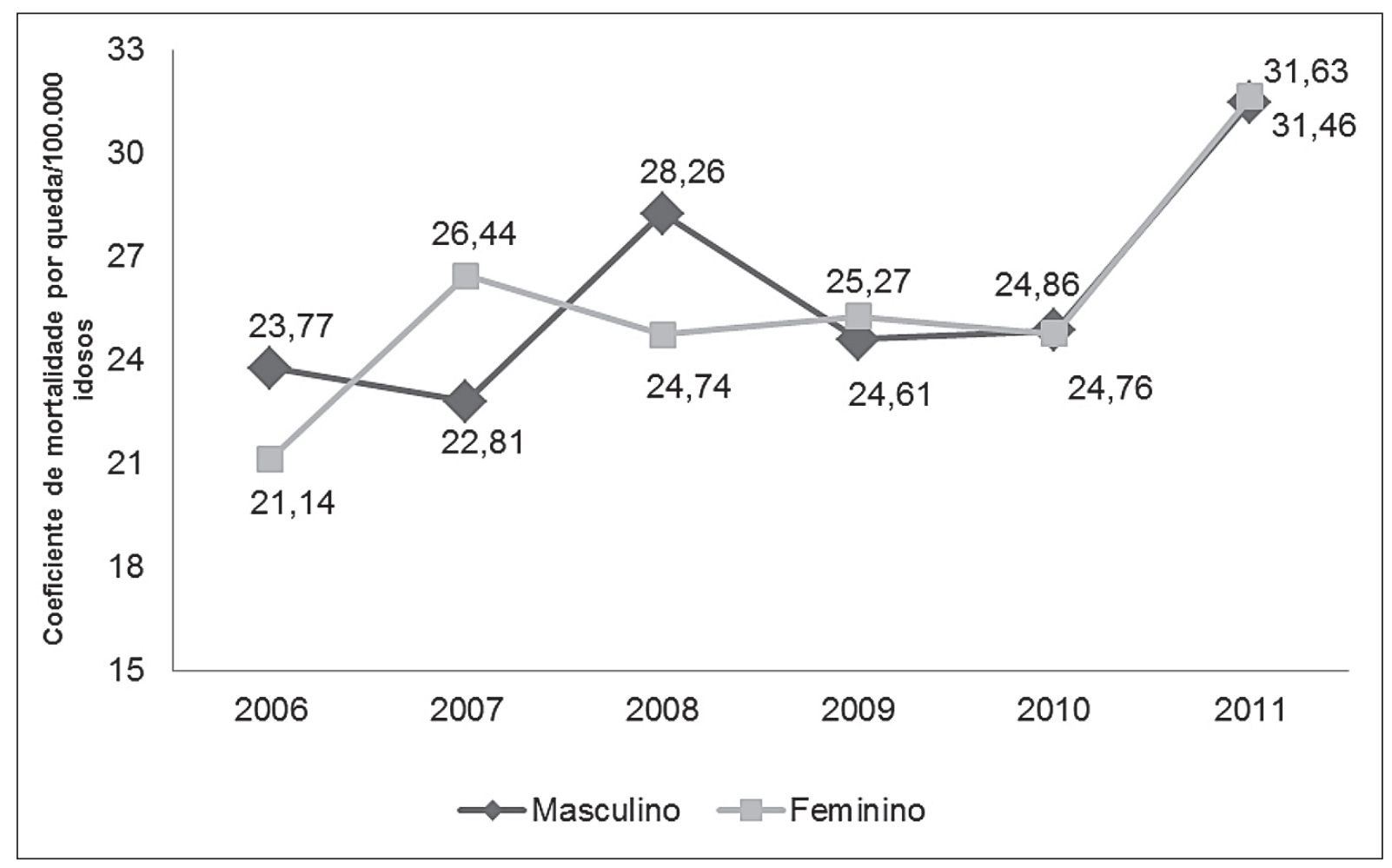

Figura 1. Coeficiente de mortalidade específico por queda em idosos, entre homens e mulheres no RS de 2006 a 2011. 
Observa-se que, para o período de estudo, houve aumento de 49,6\% no coeficiente de mortalidade específico por queda para as mulheres, enquanto que para os homens houve aumento de 32,4\%. Embora o acréscimo no período (2006 a 2011) tenha sido maior para as mulheres, verifica-se que estes coeficientes para os últimos três anos são praticante idênticos.

Na figura 2, pode-se identificar os coeficientes de mortalidade específicos por queda na população idosa, por faixa etária.

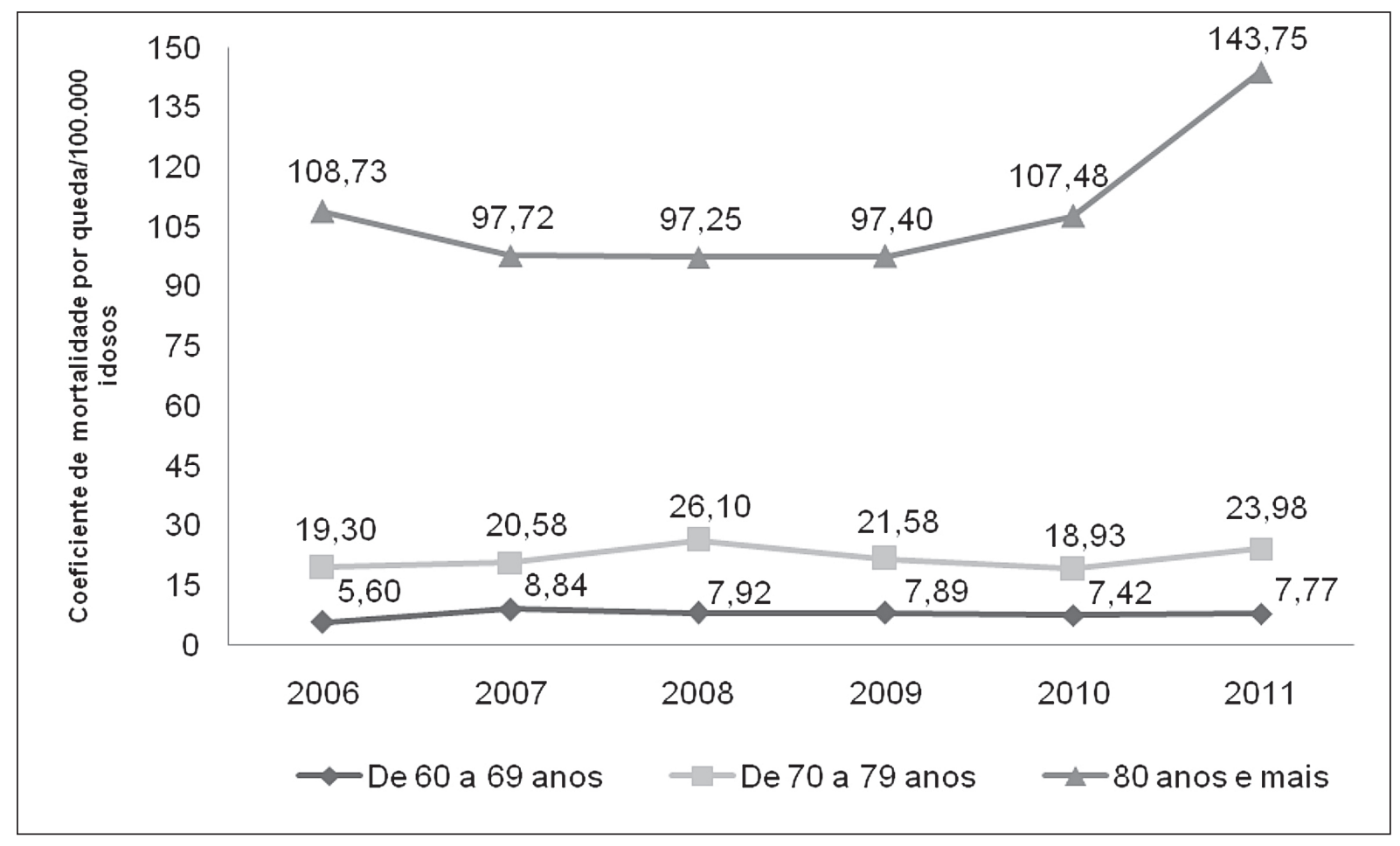

Figura 2. Coeficiente de mortalidade específico por queda em idosos, por faixa etária, no RS de 2006 a 2011.

Para a faixa etária de 80 anos ou mais, ocorre um pico no valor do coeficiente de aproximadamente 143 óbitos por queda a cada 100 mil idosos no ano de 2011, podendo-se analisar que os coeficientes para esta faixa etária são bem maiores em relação às outras. Portanto, fica claro que com o aumento da idade, o risco de óbito por queda eleva-se acentuadamente.

Os coeficientes de mortalidade específicos por queda na população idosa, segundo gênero e faixa etária, de 2006 a 2011, estão representados na figura 3. 


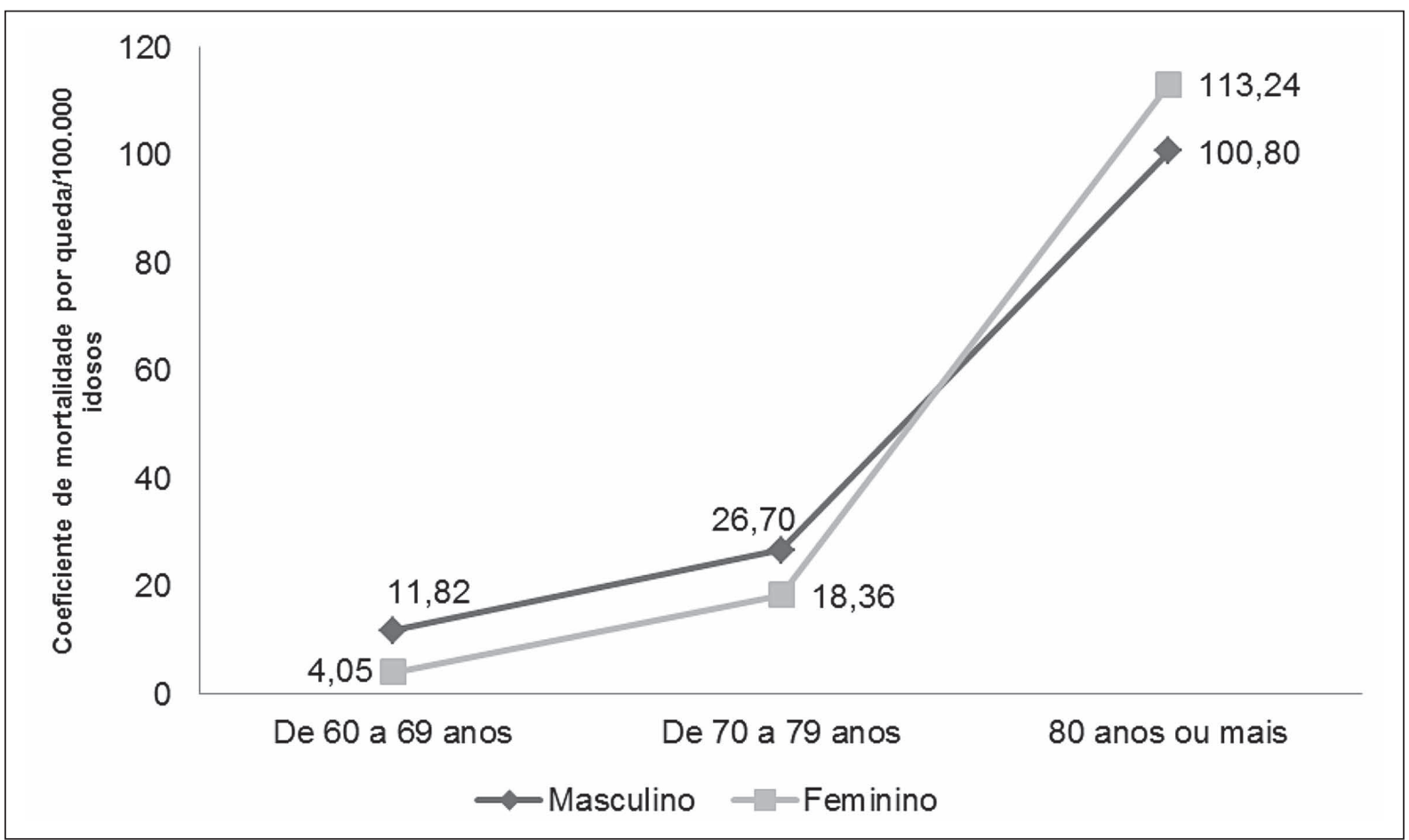

Figura 3. Coeficiente de mortalidade específico por queda em idosos, entre homens e mulheres, por faixa etária, no RS de 2006 e 2011.

Observa-se que para as faixas de idade de até 79 anos, os valores dos coeficientes são maiores para os homens; já os idosos de 80 anos ou mais, as mulheres apresentam maior coeficiente.

\section{DISCUSSÃO}

Como ponto relevante deste estudo, pode ser citado o fato de os resultados mostrarem uma perspectiva para o estado do RS como um todo a partir de fontes oficiais de informação.

No presente estudo, a maioria das mortes por quedas está relacionada ao gênero feminino $(56,9 \%)$. Em estudo realizado por Soares et al., ${ }^{15}$ em Cuiabá-MT, foi encontrada prevalência de $71,9 \%$ de idosas nas quedas, ${ }^{15}$ sendo que, essa prevalência de quedas por gênero apresenta bastante variação. Em outro estudo com idosos do estado de São Paulo, o autor encontrou 48,8\% de idosos do gênero feminino nos óbitos por queda. ${ }^{8}$ Soares et $a .^{15}$ sugerem que o declínio físicofuncional, o distúrbio motor e as consequências das doenças crônicas são diferentes entre homens e mulheres, contribuindo para um aumento do risco de quedas em mulheres comparadas aos homens.

Em relação à idade, nesta pesquisa, idosos com 80 anos ou mais possuíam maior risco de óbito por queda, sendo que nessa faixa etária, o idoso 
tem 133\% mais chance de ocorrência de óbito $(O R=2,33)$ por queda em relação aos idosos de 60 a 69 anos. Ao analisar a queda nos idosos com 80 anos ou mais de idade, Aguiar \& Assis ${ }^{26}$ e Pereira et al. ${ }^{27}$ encontraram, respectivamente, $29,6 \%$ e 20,8\%. Possivelmente, fatores como maior oscilação da marcha, fragilidade e incapacidade funcional, bem como maior número de doenças crônicas, podem estar envolvidos com a ocorrência de quedas em idades avançadas. ${ }^{28}$

No que se refere à cor da pele, evidenciou-se maior risco de óbito por queda para a cor de pele branca, indo ao encontro de estudos em que a cor da pele branca esteve relacionada com a queda, mas não com o óbito por queda. ${ }^{29,30}$ Segundo o Censo Demográfico de 2010, no estado do Rio Grande do Sul, 83,2\% da população era da cor branca. $^{31}$

Em relação ao estado civil, a prevalência de viúvos nos óbitos por queda foi de 48,5\%, sendo o risco de mortalidade por queda maior para os viúvos $(O R=1,42)$. Considerando estudos sobre quedas em idosos viúvos, dos que caíram, $45 \%{ }^{17}$ residiam em Juiz de Fora-MG e 44,8\% $0^{30}$ em Pelotas-RS. Segundo Siqueira et al., o fato de morar sozinho, que ocorre com maior frequência nessa condição, poderia delegar ao idoso tarefas que, associadas à instabilidade postural, causariam maior número de situações de risco para quedas.

Quanto à escolaridade, constatou-se que dos idosos que foram a óbito por queda, a maioria tinha de um a três anos de estudo (40,6\%). Porém, não houve diferença significativa nos riscos de óbitos por queda em função da escolaridade. No estudo de Paula et al., ${ }^{1}$ a maioria dos idosos internada por queda possui escolaridade até o primário incompleto. Pereira et al. ${ }^{27}$ afirmam que idosos com mais anos de estudo possuem menor risco para quedas, fato que pode estar relacionado a maior renda em idosos com maior escolaridade, e consequentemente, melhores condições de moradia e acesso à saúde, o que não foi comprovado no presente estudo para óbitos por queda.
Encontrou-se, nesta pesquisa, aumento do número de idosos na população nos anos estudados, no percentual de óbitos por queda e, consequentemente, nos coeficientes de mortalidade específicos por queda. $\mathrm{O}$ crescimento no coeficiente pode ser explicado por um aumento real e/ou pela mudança ocorrida em 2011, no conteúdo das DOs, com maior detalhamento das informações coletadas. ${ }^{24}$ Além disso, esses números destacam a importância de estudos na área e corroboram as implicações políticas, sociais e sobre o próprio sistema de saúde, decorrentes do processo de envelhecimento populacional. Estudo realizado com idosos em Minas Gerais verificou valores de coeficientes de mortalidade por quedas e outras causas externas de lesões acidentais superiores ao encontrado na presente pesquisa (2006 a 2008), pois considerou, além das quedas, outras causas externas. $^{2}$

Outra característica encontrada no presente estudo foi que, no período de 2006 a 2008, houve oscilação dos valores dos coeficientes para ambos os gêneros. A partir de 2009, estes se aproximaram, havendo acréscimo importante em 2011. Estudo ${ }^{2}$ também demonstra flutuação discreta dos valores do coeficiente de mortalidade por quedas e outras causas externas de lesões acidentais nos anos de 2006 a 2008, sendo as quedas as principais causas de óbito observadas. Em estudo ${ }^{8}$ realizado em São Paulo, em 2007, é possível verificar que os homens apresentaram valores superiores de coeficientes de mortalidade por queda $(36,4 / 100 \mathrm{mil})$ ao encontrado na presente pesquisa $(22,81 / 100 \mathrm{mil})$. Como forma de redução dos coeficientes de mortalidade por queda, autores apontam que os idosos precisam ser mais ativos e independentes. ${ }^{1}$

Quanto aos coeficientes de mortalidade por queda por faixas etárias, pode-se comprovar que idosos mais velhos possuem maior chance de morrer por queda. Foi constatado, neste estudo, para o ano de 2011, um coeficiente superior de mortalidade por queda (143,75/100 mil idosos) para os idosos com 80 anos ou mais de idade em relação à pesquisa realizada nos Estados 
Unidos, que obteve 107,6/100 mil, ${ }^{20}$ e ao estudo de Gawryszewski, ${ }^{8}$ que encontrou 110,7/100 $\mathrm{mil}^{8}$ idosos residentes no estado de São Paulo. Segundo Cruz et al., ${ }^{17}$ o processo de envelhecimento acarreta alterações estruturais e funcionais que se acumulam de forma progressiva com o avançar da idade, comprometendo o desempenho de atividades motoras e a adaptação ao ambiente, contribuindo para a ocorrência da queda.

Considerando o gênero e a faixa etária, foi encontrado, neste estudo, que os homens apresentaram maiores coeficientes de mortalidade por queda do que as mulheres para as faixas de 60 a 79 anos. Somente na faixa de 80 anos ou mais é que as mulheres se sobrepõem aos homens. Os coeficientes aumentam com o aumento da idade dos idosos, atingindo o valor de 113,24 óbitos para cada 100 mil idosos de 80 anos ou mais. Resultado semelhante foi encontrado no estado de São Paulo, em 2007, por outro autor para essa faixa de idade $(115,8 / 100$ mil idosos $){ }^{8}$

Em estudo ${ }^{2}$ sobre mortalidade por quedas em idosos realizado em Minas Gerais, os autores encontraram aumento nos coeficientes de mortalidade por queda para os idosos de 14,3/100 mil para 27,4/100 mil, e para as idosas, de 9,4/100 mil para 19,1/100 mil no ano de 2000 para 2007. Com isso, o risco de morrer em decorrência de quedas para essa região e período dobrou entre os idosos.

As explicações para diferenças entre os gêneros são controversas. Alguns fatores podem ser considerados, tais como: as mulheres podem apresentar menor quantidade de massa magra e de força muscular em relação aos homens da mesma idade; maior perda de massa óssea, devido à redução de estrógeno, aumentando a possibilidade de osteoporose e consequentes fraturas; maior ocorrência de doenças crônicas; maior exposição a atividades domésticas e a alterações emocionais. ${ }^{17,32}$

A descrição da mortalidade por queda em idosos aqui apresentada revela a importância de novos modelos assistenciais capazes de melhorar a relação custo-benefício da prevenção, da detecção precoce e do tratamento das morbidades associadas à ocorrência de quedas. Sendo assim, é de suma importância conhecer o perfil da população idosa que apresentou queda, com o objetivo de instrumentalizar os profissionais para aprimorar as práticas assistenciais. Mesmo compreendendo a complexidade de fatores envolvidos com as causas externas de mortalidade, é preciso questionar o impacto das medidas governamentais diante do crescimento observado.

Além de medidas de cuidados e proteção assumidas desde os níveis primários de atenção à saúde, a identificação de marcadores que aumentam a possibilidade dos eventos de queda e a melhoria no ambiente físico residencial da população idosa devem ser o ponto de partida de prevenção de agravos, proporcionando a redução dos coeficientes de mortalidade por queda em idosos.

Destaca-se que as limitações do presente estudo podem estar relacionadas à utilização de dados secundários, à cobertura e à qualidade da informação, que limitam o número de variáveis que poderiam ser relevantes para a análise. Quanto ao SIM, este sofre influência em função da melhoria da qualidade das informações registradas nas DOs. Portanto, os óbitos cujas causas básicas eram registradas em "outras causas externas" ou "acidentes de natureza não especificada" ou, ainda, por "outros acidentes de transporte", estariam agora sendo mais bem definidas, melhorando assim a qualidade das informações registradas nas DOs.

\section{CONCLUSÃO}

Entre os anos de 2006 e 2011, os óbitos por quedas representaram $0,69 \%$ em relação ao total de óbitos no Rio Grande do Sul. A chance de óbitos por queda nos idosos é significativamente maior para o gênero feminino, para a faixa etária acima de 69 anos, para idosos com cor de pele branca e viúvos ou solteiros. A escolaridade não esteve associada com a ocorrência de óbitos por quedas. 
A constatação do aumento da população de idosos no Rio Grande do Sul, no período de estudo, não difere de outras populações. $\mathrm{O}$ importante foi constatar que os coeficientes de mortalidade por quedas aumentaram nesse período, chegando a aproximadamente 32 óbitos por queda a cada 100 mil idosos no ano de 2011 - resultado de relevância para o desenvolvimento de políticas públicas para o idoso.

\section{REFERÊNCIAS}

1. Paula FL, Da Fonseca MJM, De Oliveira RVC, Rozenfeld S. Perfil de idosos com internação por quedas nos hospitais públicos de Niterói (RJ). Rev Bras Epidemiol 2010;13(4):587-95.

2. Gomes LMX, Barbosa TLA, Caldeira AP. Envelhecimento e longevidade no Rio Grande do Sul: um perfil histórico, étnico e de morbimortalidade dos idosos. Esc Anna Nery Rev Enferm 2010;4(4):779-86.

3. Magalhães APR, Paiva SC, Ferreira LOC, Aquino TA. A mortalidade de idosos no Recife: quando o morrer revela desigualdades. Epidemiol Serv Saúde 2011;20(2):183-92.

4. Instituto Brasileiro de Geografia e Estatística [Internet]. Brasília, DF: IBGE; [1995] - . Pesquisa Nacional por Amostra de Domicílios 2010; 2010 [acesso 10 fev 2015]; [aproximadamente 2 telas]. Disponível em: http://www.ibge.gov.br/ home/estatistica/populacao/condicaodevida/ indicadoresminimos/ sinteseindicsociais2010/ SIS_2010.pdf.

5. Messias MG, Neves RF. A influência de fatores comportamentais e ambientais domésticos nas quedas em idosos. Rev Bras Geriatr Gerontol 2009;12(2):27582.

6. Goottlieb MGV, Schwanke CHA, Gomes I, Da Cruz IBM. Envelhecimento e longevidade no Rio Grande do Sul: um perfil histórico, étnico e de morbimortalidade dos idosos. Rev Bras Geriatr Gerontol 2011;14(2):365-80.

7. Siqueira FV, Facchini LA, Silveira DS, Piccini RX, Tomasi E, Thumé E, et al, Prevalence of falls in elderly in Brazil: a countrywide analysis. Cad Saúde Pública 2011;27(9):1819-26.

8. Gawryszewski VP. A importância das quedas no mesmo nível entre idosos no estado de são Paulo. Rev Assoc Med Bras 2010;56(2):162-7.

9. Centers for Disease Control and Prevention [Internet]. Atlanta: CDC; 2003. Web-based Injury
É importante salientar que os coeficientes de mortalidade por queda são extremamente mais elevados para a faixa etária de 80 anos ou mais, e que os coeficientes de uma maneira geral aumentaram no último ano do estudo, indicando aumento real do numero de óbitos por queda e/ ou a melhoria nos registros da causa básica de óbito.

Statistics Query and Reporting System (WISQARS); 2003 [acesso em 28 Ago 2014] [aproximadamente 2 telas]. Disponível em: http://www.cdc.gov/ homeandrecreationalsafety/falls/adultfalls.html

10. Ziade N, Jougla E, Coste J. Using vital statistics to estimate the population-level impact of osteoporotic fractures on mortality based on death certificates, with an application to France (2000-2004).BMC Public Health 2009;9:1-14.

11. Gasparotto LPR, Falsarella GR, Coimbra AMV. As quedas no cenário da velhice: conceitos básicos e atualidades da pesquisa em saúde. Rev Bras Geriatr Gerontol 2014;17(1):201-9.

12. Nascimento BN, Duarte BV, Antonini DG, Borges SM. Risco para quedas em idosos da comunidade: relação entre tendência referida e susceptibilidade para quedas com o uso do teste clínico de interação sensorial e equilíbrio. Rev Soc Bras Clin Méd 2009;7:95-9.

13. Monteiro CR, Faro ACM. Avaliação funcional de idoso vítima de fraturas na hospitalização e no domicílio. Rev Esc Enferm USP 2010t;44(3):719-24.

14. Meireles AE, Pereira LMS, De Oliveira TG, Christofoletti G, Fonseca AL. Alterações neurológicas fisiológicas ao envelhecimento afetam o sistema mantenedor do equilíbrio. Rev Neurocienc 2010;18(1):103-8.

15. Soares WJS, De Moraes SA, Ferriolli E, Perracini MR. Fatores associados a quedas e quedas recorrentes em idosos: estudo de base populacional. Rev Bras Geriatr Gerontol 2014;17(1):49-60.

16. Stevens JA, Sogolow ED. Preventing Falls: What Works. A CDC Compendium of Effective Community-based Interventions from Around the World. Atlanta: National Center for Injury Prevention and Control; 2008.

17. Cruz DT, Ribeiro LC, Vieira MT, Teixeira MTB, Bastos RR, Leite ICG. Prevalência de quedas e fatores associados em idosos. Rev Saúde Pública 2012;46(1):138-46. 
18. Rossini M, Mattarei A, Braga V, Viapiana O, Zambarda C, Benini C, et al. Risk factors for hip fracture in elderly persons. Reumatismo 2010;62(4):273-82.

19. Rodrigues IG, Fraga GP, Barros MBA. Quedas em idosos: fatores associados em estudo de base populacional. Rev Bras Epidemiol 2014;705-18.

20. Centers for Disease Control and Prevention [Internet]. Atlanta: National Center for Injury Prevention and Control. [1997]; [atualizado $11 \mathrm{fev}$ 2015; acesso em 28 Ago 2014]. Disponível em: http:// www.cdc.gov/ncipc/wisqars.

21. Lopes KT, Costa DF, Santos LF, Castro DP, Bastone AC. Prevalência do medo de cair em uma população de idosos da comunidade e sua correlação com mobilidade, equilíbrio dinâmico, risco e histórico de quedas. Rev Bras Fisioter 2009;13(3):223-9.

22. Organização Mundial da Saúde, Centro Brasileiro de Classificação de Doenças em Português. Classificação Estatística Internacional de Doenças e Problemas Relacionados à Saúde. 10ª rev. São Paulo: EDUSP; 1998.

23. Secretaria Estadual de Saúde, Núcleo de Informações em Saúde. Sistema de informação sobre mortalidade no Rio Grande do Sul [Internet]. Porto Alegre: Secretaria Estadual de Saúde; [atualizado em 25 Fev 2015; acesso em 21 Set. 2014]. Disponível em: http://www.saude.rs.gov.br/wsa/portal/ index. jsp?menu= organograma $\& \operatorname{cod}=746$.

24. Portal da Saúde. DATASUS [Internet]. Brasília, DF: Ministério da Saúde; c2008- . Informações de saúde demográficas e socioeconômicas. Projeções intercensitárias; [acesso em 24 jun 2014]; [aproximadamente 1 tela]. Disponível em: http:// tabnet.datasus.gov.br
25. Brasil. Ministério da Saúde. Estatuto do Idoso [Internet]. Brasília, DF: Ministério da Saúde; 2003. [Acesso em 24 jun 2013]. Disponível em: http:// bvsms.saude.gov.br/bvs/publicacoes/estatuto_idoso. pdf.

26. Aguiar CF, Assis M. Perfil de mulheres idosas segundo a ocorrência de quedas: estudo de demanda no Núcleo de Atenção ao Idoso da UnATI/UERJ. Rev Bras Geriatr Gerontol 2009;12(3):391-404.

27. Pereira GN, Morsch P, Lopes DGC, Trevisan MD, Ribeiro A, Navarro JHN, et al. Fatores socioambientais associados à ocorrência de quedas em idosos. Ciênc Saúde Coletiva 2013,18(12):3507-14.

28. Deandrea S, Lucenteforte E, Bravi F, Foschi R, La Vecchia C, Negri E. Risk factors for falls in community-dwelling older people: a systematic review and meta-analysis. Epidemiology 2010;21(5):658-68.

29. Gonçalves LG, Vieira ST, Siqueira FV, Hallal PC. Prevalência de quedas em idosos asilados do município de Rio Grande, RS. Rev Saúde Pública 2008;42(5):1-8

30. Carvalho MP, Luckow ELT, Siqueira FV. Quedas e fatores associados em idosos institucionalizados no município de Pelotas (RS, Brasil). Ciênc Saúde coletiva 2011;16(6):2945-52.

31. Instituto Brasileiro de Geografia e Estatística. População total e respectiva distribuição percentual, por cor ou raça, segundo as grandes regiões, unidades da federação e Regiões Metropolitanas: Síntese dos Indicadores Sociais 2008 [Internet]. Rio de Janeiro: IBGE; [1995] - [Acesso 2013 set 18]. Disponível em: http://www.ibge.br.

32. Perracini MR, Ramos LR. Fatores associados a quedas em uma coorte de idosos residentes na comunidade. Rev Saúde Pública 2002;36(6): 709-16. 